\title{
Novel In-Vivo Experimental Analyses in Support of Determination if the Success of Treatment
}

\section{Jacek MK*}

Department of Pathology and Molecular Medicine, McMaster University, Hamilton, Ontario, Canada

*Corresponding author: Jacek M Kwiecien, Department of Pathology and Molecular Medicine, McMaster University, Hamilton, Ontario, Canada, Tel: 905-525-

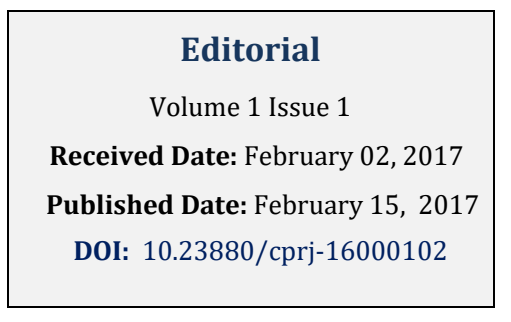

9140/22827; Email: Kwiecien@mcmaster.ca.

\section{Editorial}

It is an honor and privilege to contribute an editorial to Clinical Pathology and Research Journal and we hope to become a recognized medium for laboratory clinicians and medical researchers and attract quality manuscripts dealing with an important issue of clinical pathology.

In my research activities, often serving as a collaborating experimental neuropathologist, I often asked a question; do weunderstand the pathogenesis of the disease we are studying? And then the next one; what are in vivo analytic methods that can be used to first study the progression of the disease during its course and then to monitor the effect of an experimental therapy? Keeping in mind feasible adaptation of such in vivo methods to clinical studies in patients.

Stroke, traumatic brain injury (TBI) and spinal cord injury (SCI) are a group of incurable clinical entities that constitute arguably the most devastating and the most important numerically pathogenesis facing medicine. The understanding of the pathogenesis remains poor and attempts at substantially limiting neurological deficits with experimental therapies have not been successful. Systematic studies of suitable experimental animal models at the level of anatomic pathology and clinical pathology are lacking.

Preliminary studies indicate that experimental SCI in a rat model leads to a remarkably severe inflammatory process of unusually long duration and tissue-destructive action. Pilot studies using powerful anti-inflammatory steroids infused directly into the site of injury and inflammation indicate that anti-inflammatory compounds less toxic than steroids may be a way to limit the destruction of the spinal cord tissue in SCI and of the brain tissue in TBI or in stroke and thus achieve significantly better clinical outcomes and improved quality of life. But the evidence supporting such optimism comes from histological analysis of the injured and treated spinal cord tissue from experimental animals.

We do not have the luxury of performing histology on human patients in future clinical trials. Furthermore, MRI or CT scans of the site of spinal injury in post-operative patients are typically of poor quality due to a severe optical blast from the metal screws nearby used to reduce the fractured vertebral bones. Therefore, how are we going to monitor the success of an anti-inflammatory therapy in an SCI patient? The current published experimental research does not tackle this issue in a satisfactory fashion and analytical tests are not available to clinical pathologists. On the other hand, meaningful progress in treating SCI, TBI and stroke will critically depend on the availability of key in vivo analytical assays to monitor progression of important elements of pathogenesis in longitudinal studies. Of course, once the pathogenesis of this group of diseases has become properly studied elucidated, understood and effective, non-toxic anti-inflammatory compound become available.

In this short editorial, using the spinal cord injury as an example, I am hoping to turn the attention of the readers 


\section{Clinical Pathology \& Research Journal}

and contributors including pathologists, clinicians and 2. Kwiecien JM, Jarosz B, Oakden W, Klapec M, Stanisz GJ, et medical researchers to the importance of clinical pathology first in experimental research and ultimately to individual patients. After all it is good to consult the wisdom of former generations:

\section{References}

1. Kwiecien JM, Jarosz B, Machova UL, Rola R, Dabrowski W (2015) Subdural infusion of dexamethasone inhibits leukomyelitis after acute spinal cord injury in a rat model.

Folia Neuropathol 53(1): 41-51.

3. Oakden W, Kwiecien JM, O Reilly MA, Lake E, Akens MK, et al. (2014) A non-surgical model of cervical spinal cord injury induced with focused ultrasound and microbubbles. J Neurosci Methods 235: 92-100. al. (2016) An in vivo model of anti-inflammatory activity of subdural dexamethasone following the spinal cord injury. Neurol Neurosurg Pol 50(1): 7-15.

- J Neurosci Methods 235: 92-100. 